\title{
CARACTERIZAÇÃO DOS CASOS DE DENGUE POR LOCALIZAÇÃO NO INTERIOR DE MATO GROSSO ENTRE 2007 E 2016
}

\author{
Débora Aparecida da Silva Santos ${ }^{1}$, Anne Caroline Farias Ribeiro Freitas ${ }^{2}$, Érica Regina de Melo Panhan ${ }^{2}$, \\ Ricardo Alves de Olinda ${ }^{3}$, Letícia Silveira Goulart ${ }^{4}$, Valéria Cristina Menezes Berredo ${ }^{1}$
}

\begin{abstract}
RESUMO: Objetivo: caracterizar os casos notificados de dengue de acordo com a localização, em Rondonópolis, Mato Grosso, entre 2007 e 2016. Método: estudo epidemiológico do tipo ecológico de abordagem quantitativa e descritiva. Dados de fonte secundária incluindo variáveis sociodemográficas, clínicas e localização dos casos de dengue. Foi utilizada estatística descritiva e apresentados mapas dos quantis dos casos, bem como a distribuição por bairros. Resultados: há indicativos que, embora seja observada notificação por toda extensão territorial, proliferou principalmente no Centro, Centros A e B e Vila Aurora, considerados bairros populosos e povoados. Também se observou que áreas com maior número de casos estão localizadas próximas a rios, córregos e áreas ambientais protegidas. Conclusão: o retrato geográfico da dengue deve alertar pesquisadores e a gestão de saúde do município sobre o comportamento da doença, a fim de um planejamento eficiente e execução de ações de prevenção e controle.

DESCRITORES: Dengue; Distribuição espacial da população; Infecções por arbovírus; Promoção da saúde; Atenção Primária a Saúde.

\section{CHARACTERIZATION OF DENGUE CASES BY LOCATION IN RURAL MATO GROSSO STATE BETWEEN 2007 AND 2016}

\begin{abstract}
Objective: to characterize the reported dengue cases according to location, in Rondonópolis, Mato Grosso, between 2007 and 2016. Method: epidemiological, ecological, quantitative study using a descriptive approach. Secondary source data including sociodemographic and clinical variables and location of dengue cases. Descriptive statistics were used and maps of the quantiles of the cases were presented, as well as the distribution by neighborhoods. Results: there were indications that, although notifications were observed over the entire area, the disease mainly proliferated in Centro, Centros A and B and Villa Aurora, considered populous neighborhoods. It was also observed that areas with greater numbers of cases were located near rivers, streams and areas of environmental protection. Conclusion: the geographic picture of dengue should warn researchers and the health management of the municipality about the behavior of the disease, aiming for efficient planning and implementation of prevention and control actions.
\end{abstract}

DESCRIPTORS: Dengue; Spatial distribution of population; Arbovirus infections; Health promotion; Primary Health Care.

\section{CARACTERIZACIÓN CASOS DE DENGUE POR LOCALIZACIÓN EN INTERIOR DE MATO GROSSO ENTRE 2007 Y 2016}

RESUMEN: Objetivo: Caracterizar los casos notificados de dengue según su localización en Rondonópolis, Mato Grosso, entre 2007 y 2016. Método: Estudio epidemiológico del tipo ecológico, con abordaje cualitativo y descriptivo. Datos de fuente secundaria, incluyendo variables sociodemográficas, clínicas y localización de los casos de dengue. Se aplicó estadística descriptiva, se presentaron mapas por porcentual de casos y de distribución por barrios. Resultados: Se presume que, aunque se observen notificaciones en toda la extensión territorial, proliferó principalmente en el Centro, Centros A y B y Vila Aurora, considerados barrios populosos y poblados. También se observó que las áreas con mayor número de casos están localizadas cerca de ríos, arroyos y áreas ambientales protegidas. Conclusión: El esquema geográfico del dengue debe alertar a investigadores y a la gestión sanitaria municipal sobre el comportamiento de la enfermedad, para una planificación eficiente y la ejecución de acciones de prevención y control.

DESCRIPTORES: Dengue; Distribución Espacial de la Población; Infecciones por Arbovirus; Promoción de la Salud; Atención Primaria de Salud.

${ }^{1}$ Enfermeira. Doutora em Recursos Naturais. Docente de Enfermagem da Universidade Federal de Mato Grosso. Rondonópolis, MT, Brasil.

²Enfermeira. Universidade Federal de Mato Grosso. Rondonópolis, MT, Brasil.

${ }^{3}$ Farmacêutica. Doutora em Ciências. Docente de Enfermagem da Universidade Federal de Mato Grosso. Rondonópolis, MT, Brasil.

${ }^{4}$ Estatístico. Doutor em Estatística. Docente da Universidade Estadual da Paraíba. Campina Grande, PB, Brasil.

Autor Correspondente:

Débora Aparecida da Silva Santos

Universidade Federal de Mato Grosso

Av. dos Estudantes, 5055 - 78735-901 - Rondonópolis, MT, Brasil

E-mail: deboraassantos@hotmail.com
Recebido: $21 / 11 / 2018$

Finalizado: 18/10/2018 


\section{INTRODUÇÃO}

As arboviroses representam importantes doenças que acometem humanos e podem ocasionar grande impacto econômico na sociedade. A arbovirose de maior destaque em saúde pública no Brasil é a dengue, com crescente apresentação nos últimos anos, e consequentemente aumento de casos das formas graves da doença, devido a sua alta patogenicidade e chances de recidivas ${ }^{(1-2)}$.

A dengue é uma doença viral transmitida pela fêmea do mosquito, por um dos quatro sorotipos dos vírus, sendo conhecidos DEN-1, DEN-2, DEN-3 e DEN-4. É uma doença febril aguda causada por um arbovírus e transmitida pelo mosquito do gênero Aedes e espécie aegypt. O vírus da dengue pertence ao gênero Flavivírus da família Flaviviridae composta por 73 vírus, das quais 40 espécies estão associadas com o desenvolvimento de doenças em humanos. O mosquito se alimenta do sangue do indivíduo infectado e que se encontra em fase de viremia e o vírus aloja nas glândulas salivares do mosquito, se proliferando, infectando com sua saliva o homem ${ }^{(2-3)}$.

O gênero Aedes possui duas espécies: Aedes aegypti e Aedes albopictus. O A.aegypti é o principal transmissor da dengue e da febre amarela no meio urbano e o A.albopictus representa um vetor secundário na transmissão da dengue silvestre ${ }^{(2)}$.

É importante ressaltar que a dinâmica da ocorrência das arboviroses está intimamente relacionada a características como: condições ecológicas, sociais, climáticas e demográficas de determinada região, além da grande disponibilidade de condições favoráveis para o desenvolvimento do vetor no meio urbano, das características inerentes ao vetor como sua distribuição, competência e a especificidade da espécie. Outro fator a ser considerado são os desafios para o setor da vigilância epidemiológica que tem que intervir juntos aos meios biológicos, ambientais (inspeção e eliminação de reservatórios), com a própria população (informação, educação) e os recursos disponibilizados pelas esferas governamentais ${ }^{(4-5)}$.

A Organização Mundial da Saúde (OMS) propõe uma classificação com duas categorias: dengue grave e não grave. A ocorrência dos diferentes sorotipos, associada ao aumento significativo de casos, além das características intrínsecas das pessoas infectadas, são os principais fatores relacionados às formas graves e letais da doença. Acredita-se que a doença esteja aumentando anualmente, tornando grave problema de saúde em todo o território nacional, e para o qual atualmente não existem medidas de controle sustentáveis, capazes de parar a rápida distribuição da doença ${ }^{(6)}$.

Neste contexto, o estudo da caracterização da localização da dengue fornece informações que não seriam visualizadas trabalhando apenas com dados tabulares, percebendo-se a importância desta distribuição quando se pretende analisar a localização geográfica dos focos de dengue em períodos endêmicos, para tentar intervir de maneira a minimizar as ocorrências e na busca pela erradicação do vetor através do conhecimento de seu habitat ${ }^{(7)}$.

São escassos os estudos que elucidam onde são notificados estes casos no estado de Mato Grosso, contudo, alguns autores estudaram este tema no mundo, no Brasil e inclusive, na região CentroOeste $^{(8-10)}$. Rondonópolis, localizada no estado de Mato Grosso, apresenta extensa área geográfica e não se tem estudo e publicação da distribuição espacial real da dengue. Assim justifica-se a realização desta pesquisa, a fim de conhecer melhor o comportamento da doença, o qual é fundamental para a promoção e execução de medidas que visem o controle e suporte a ações prescritas pelos programas de controle de vetores e epidemias.

Neste contexto, esta pesquisa tem por objetivo caracterizar os casos notificados de dengue de acordo com a localização, no município de Rondonópolis-MT, no período de 2007 a 2016.

\section{MÉTODO}

O local de estudo foi o município de Rondonópolis-MT com dados relativos ao período de janeiro de 2007 a dezembro de 2016. Cabe ressaltar que a seleção desta série histórica foi devido à disponibilidade dos dados encontrados nos sites de buscas oficiais e tempo considerável para uma análise robusta 
dos dados. Somente a partir de 2001 foi instituída a obrigatoriedade da notificação compulsória e a informatização pública dos dados relativos a essas informações, sendo delimitado esse período dos últimos dez anos.

O estado de Mato Grosso possui 3.305 .531 habitantes, distribuídos em 141 municípios, com características sociais, econômicas, geográficas, demográficas, ambientais, culturais e epidemiológicas heterogêneas. Em seu último censo, no município de Rondonópolis, no ano de 2016, havia uma população estimada de 218.999 habitantes. A área da unidade territorial é equivalente a 4.159,118 km2 (sendo 129,2 km² de zona urbana e 4.029,922 km² de zona rural) e densidade demográfica 258/47,00 hab./ km2, cujo bioma é o cerrado e apresenta clima tropical úmido ${ }^{(11)}$.

Este município está localizado entre as coordenadas de $16^{\circ} 25^{\prime \prime}$ e $16^{\circ} 30^{\prime \prime} \mathrm{S}$ e $54^{\circ} 33^{\prime}$ e $54^{\circ} 39^{\prime \prime} \mathrm{W}$, distando aproximadamente $1.440 \mathrm{~km}$ (em linha reta) a oeste da costa do Oceano Atlântico. O sítio urbano, com altitudes que variam de 200 a 300 metros, ocupa topograficamente a maior parte do vale e baixas colinas do Rio Vermelho (baixo curso). O quadro físico estende-se num sítio convergente, de topografia plana a levemente ondulada da confluência (margem esquerda) dos Rios Arareau e Vermelho ${ }^{(12)}$.

Esta posição num nível morfológico rebaixado ou de depressão tem importantes implicações no clima local, na estrutura do clima urbano e no ritmo dos atributos climáticos. O entorno próximo (distâncias entre 20 e $100 \mathrm{~km}$ ) é constituído por um anfiteatro de terras altas (chapadas e serras) cujos topos variam entre 700 e 800 metros. As mais importantes: encontram-se ao sudeste a Serra da Petrovina, a noroeste a Serra de São Vicente, ao norte o planalto de Campo Verde-Primavera do Leste e ao sul a Serra da Onça ${ }^{(12)}$ (Figura 1).

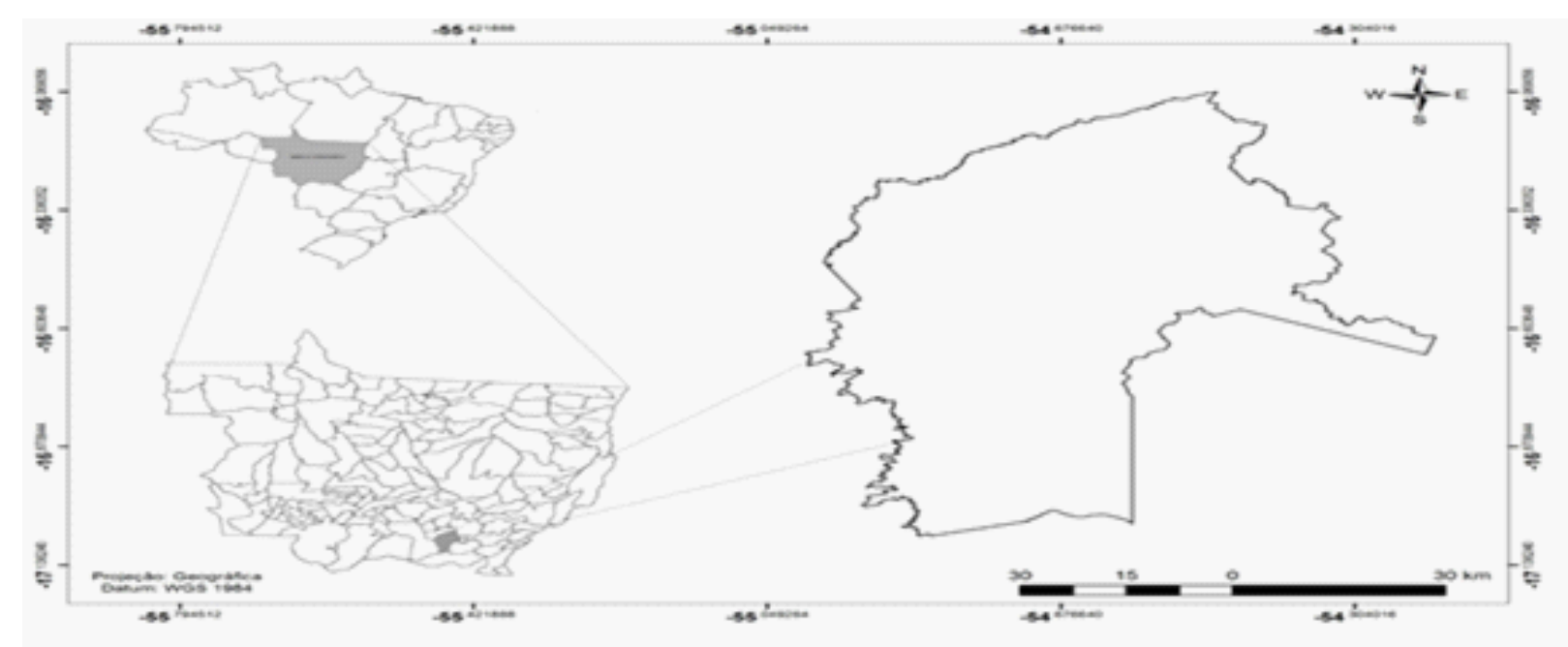

Figura 1- Localização geográfica do município de estudo. Rondonópolis, MT, Brasil, 2017

Trata-se de um estudo epidemiológico do tipo ecológico de abordagem quantitativa e descritiva. Os dados foram adquiridos de fonte secundária, relativos às séries de dados da doença de notificação compulsória do Sistema de Informação de Agravos de Notificação (SINAN), especificamente a dengue, do período que corresponde a dez anos. A ficha de investigação criada por este Sistema é composta por dados gerais (município, data da notificação e Unidade de Saúde), individuais (identificação, sexo, raça, escolaridade), da residência (endereço, zona urbana ou rural) e laboratoriais para a classificação final da evolução do caso.

Estes dados fazem parte do banco de dados de acesso público e gratuito do Departamento de Informação do Sistema Único de Saúde (DATASUS), que integra a Secretaria de Gestão Estratégica e Participativa, conforme decreto $\mathrm{n}^{\circ}$ 8.065, de 07 de agosto de 2013, que trata da Estrutura Regimental do Ministério da Saúde. 
As variáveis estudadas incluíram as sociodemográficas relativas a: sexo (masculino e feminino), faixa etária (menor de um ano, de um a 14 anos, de 15 a 30, 31 a 54, acima de 55 anos), raça (amarela, branca, indígena, pardo/negro), zona de residência (Peri urbana, rural, urbana), nível de escolaridade (fundamental, ensino médio, ensino superior e analfabeto), e as variáveis clínicas: mês de notificação (janeiro a dezembro), classificação (clássica, dengue com complicação, febre hemorrágica), casos autóctones, forma grave e óbitos, além da distribuição dos casos em conformidade com a localidade residencial dos casos notificados no município.

Inicialmente realizou-se um estudo descritivo dos casos notificados por dengue na cidade de Rondonópolis de acordo com as variáveis sociodemográficas, clínicas e de localização por bairro dos casos notificados. Os dados foram coletados entre novembro de 2016 e janeiro de 2017, usando como critério de inclusão todos os casos de dengue e as variáveis citadas no objeto de estudo, sendo excluídos os casos com notificação com dados incompletos e inconclusivos da ficha de notificação que não permitiram os diagnósticos de dengue. Os casos com variáveis não preenchidas, consideradas como campo vazio/ignorado, foram mantidos na análise.

Na sequência, foram apresentados os mapas dos quantis dos casos notificados por dengue bem como a distribuição da localização por bairros nos últimos dez anos. Nesta análise, foi considerado o número de casos mensais de dengue para cada localização no município, no período de estudo. Estes dados foram distribuídos no shapefile fornecido pela Prefeitura Municipal de Rondonópolis, que representa os dados geoespaciais em forma de vetor usado por Sistema de Informação Geográfico (SIG).

Esta pesquisa faz parte do projeto matricial intitulado "Distribuição espacial das doenças de notificação compulsória em Mato Grosso", submetido ao Comitê de Ética em Pesquisa do Hospital Júlio Muller, por meio da Plataforma Brasil sendo aprovado com parecer 1.571.782.

\section{RESULTADOS}

Foram notificados 16.653 casos de dengue em Rondonópolis (MT) no período de janeiro de 2007 a dezembro 2016, sendo que o ano de 2010 teve o maior registro de casos 4153 (24,94\%) e o menor em 2008 com $252(1,51 \%)$.

O perfil sociodemográfico dos casos de dengue nesta pesquisa caracterizou-se por predominarem $8728(52,41 \%)$ casos do sexo feminino, $5681(34,11 \%)$ na faixa etária de 31 a 54 anos, 5810 (34,90\%) da raça branca, $16018(96,20 \%)$ residentes na zona urbana e 3305 (19,85\%) com nível de escolaridade ensino médio (Tabela 1).

Tabela 1 - Distribuição da prevalência de casos de dengue segundo variáveis sociodemográficas - 2007 a 2016. Rondonópolis, MT, Brasil, 2017 (continua)

\begin{tabular}{llcc} 
VARIÁVEIS & PERFIL & $\mathbf{N}$ & $\mathbf{\%}$ \\
\hline \multirow{3}{*}{ Sexo } & Masculino & 7923 & 47,58 \\
\cline { 2 - 4 } & Feminino & 8728 & 52,41 \\
\cline { 2 - 4 } & *Vazio/ignorado & 2 & 0,01 \\
\hline \multirow{5}{*}{ Faixa etária } & Menor de 1 ano & 588 & 3,52 \\
\cline { 2 - 4 } & De 1 a 14 anos & 4230 & 25,4 \\
\cline { 2 - 4 } & De 15 a 30 anos & 4444 & 26,69 \\
\cline { 2 - 4 } & De 31 a 54 anos & 5681 & 34,11 \\
\cline { 2 - 4 } & Acima de 55 anos & 1709 & 10,27 \\
\cline { 2 - 4 } & *Vazio/ignorado & 1 & 0,01 \\
\hline
\end{tabular}




\begin{tabular}{llcc}
\hline \multirow{4}{*}{ Raça } & Amarela & 159 & 0,95 \\
\cline { 2 - 4 } & Branca & 5810 & 34,9 \\
\cline { 2 - 4 } & Indígena & 39 & 0,23 \\
\cline { 2 - 4 } & Pardo/negro & 5437 & 32,64 \\
\cline { 2 - 4 } *Vazio/ignorado & 5208 & 31,28 \\
\hline \multirow{3}{*}{ Zona de Residência } & Periurbana & 54 & 0,32 \\
\cline { 2 - 4 } & Rural & 327 & 1,96 \\
\cline { 2 - 4 } & Urbana & 16018 & 96,2 \\
\cline { 2 - 4 } Nível de escolaridade & Fundamental & 254 & 1,52 \\
\cline { 2 - 4 } & Ensino médio & 3049 & 18,31 \\
\cline { 2 - 4 } & Ensino superior & 3305 & 19,85 \\
\cline { 2 - 4 } & Analfabeto & 935 & 5,61 \\
\cline { 2 - 4 } & *Vazio/ignorado & 9244 & 55,51 \\
\hline
\end{tabular}

Segundo as variáveis clínicas, os meses de maior notificação foram janeiro com 3495 (21\%), fevereiro 2806 (16,85\%) e março 2231 (13,40\%). Quanto à classificação da dengue, prevaleceu 7708 (46,30\%) casos de dengue clássica, contudo, $8690(52,18 \%)$ na lacuna da informação vazio/ignorado destaca-se na maioria das fichas de notificação. Foram notificados 255 (1,53\%) casos na forma da dengue grave, 16 $(0,09 \%)$ óbitos e $2520(15,13 \%)$ casos autóctones conforme exposto na Tabela 2.

Tabela 2 - Distribuição da prevalência de casos de dengue segundo variáveis clínicas, de 2007 a 2016. Rondonópolis, MT, Brasil, 2017

\begin{tabular}{llcc}
\hline \multirow{5}{*}{ VARIÁVEIS } & PERFIL & $\mathbf{N}$ & $\mathbf{\%}$ \\
\hline & Janeiro & 3495 & 21 \\
\cline { 2 - 4 } & Fevereiro & 2806 & 16,85 \\
\cline { 2 - 4 } & Março & 2231 & 13,4 \\
\cline { 2 - 4 } Mês notificado & Abril & 1592 & 9,55 \\
\cline { 2 - 4 } & Maio & 1841 & 11,05 \\
\cline { 2 - 4 } & Junho & 1153 & 6,92 \\
\cline { 2 - 4 } & Julho & 584 & 3,5 \\
\cline { 2 - 4 } & Agosto & 389 & 2,33 \\
\cline { 2 - 4 } & Setembro & 289 & 1,73 \\
\cline { 2 - 4 } & Outubro & 382 & 2,3 \\
\cline { 2 - 4 } & Novembro & 597 & 3,6 \\
\cline { 2 - 4 } & Dezembro & 7294 & 7,77 \\
\hline \multirow{3}{*}{ Classificação } & Dengue clássica & 255 & 1,53 \\
\cline { 2 - 4 } & Forma grave & 8690 & 52,18 \\
\cline { 2 - 4 } & *Vazio/ignorado & 16 & 0,09 \\
\hline Casos autóctones & & & 15,13 \\
\hline Óbito & & 2520 &
\end{tabular}


Quanto à localização, houve maior ocorrência de dengue no Centro com 990 (5,94\%) notificações e $530(3,18 \%)$ na Vila Aurora, considerados como bairros populosos e povoados, confirmando a relação do Aedes aegypti com ambientes urbanizados. Nas Figuras $2 \mathrm{a}$ e $2 \mathrm{~b}$, é possível observar que as áreas azuis possuem maior número de casos registrados no período e estão localizadas próximas a rios e córregos, assim como a áreas ambientais protegidas e com terrenos baldios ou loteamentos não habitados, tornando-se lugares com mato e propensos ao acúmulo de lixo por parte dos próprios moradores.
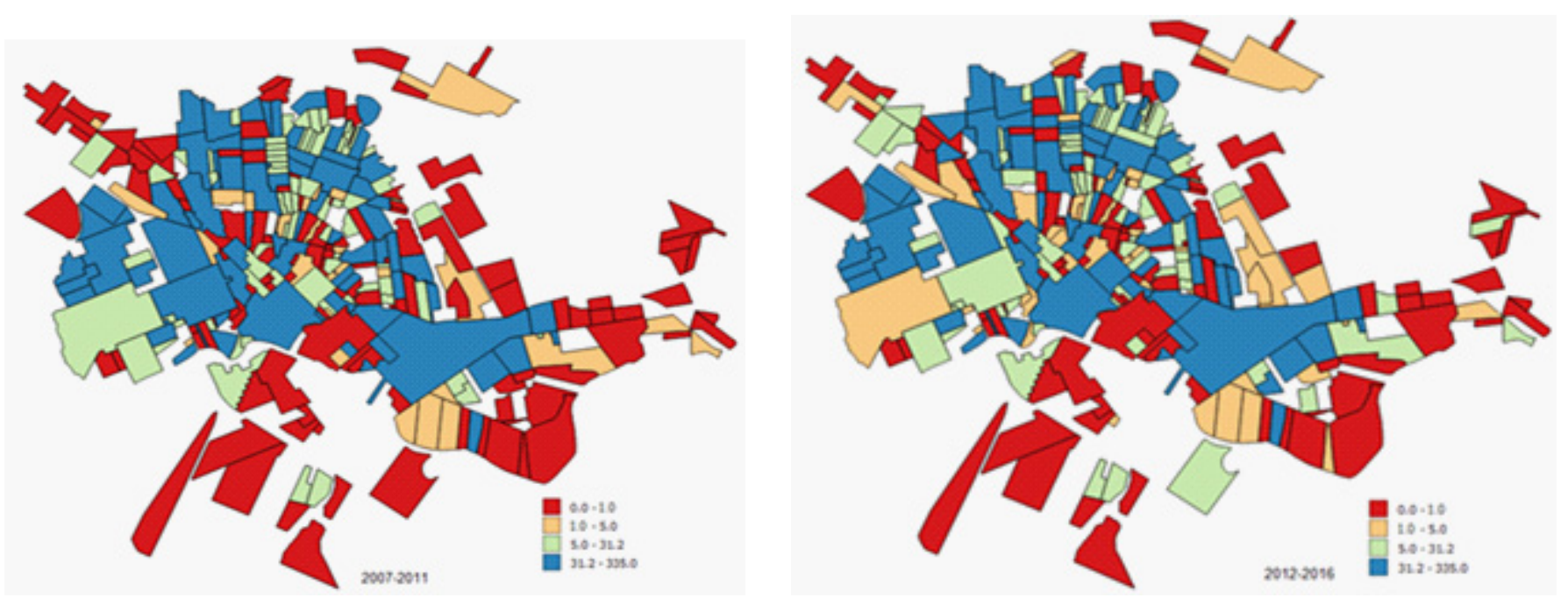

Figura 2-2a. Distribuição espacial da dengue do total de casos por bairro entre os anos 2007 a 2011 , segundo os dados encontrados no SINAN. 2b. Distribuição espacial do total de casos por bairro entre os anos 2012 a 2016 segundo notificação do SINAN. Rondonópolis, MT, Brasil, 2017.

Estes casos correspondem aos bairros 403 (2,42\%) no Conjunto São José, 493 (2,96\%) no Parque Universitário, 342 (2,05\%) no Jardim Tropical, 327 (1,96\%) no Jardim Iguaçu, 292 (1,75\%) no Monte Líbano, 293 (1,76\%) no Bairro Santa Cruz, 253 (1,52\%) na Vila Operária, 243 (1,46\%) no Cidade Alta, $222(1,33 \%)$ no Jardim Carlos Bezerra, 346 (2,07\%) no Jardim Liberdade, 217 (1,3\%) na Vila Olinda, 210 $(1,26 \%)$ no Parque Sagrada Família, $252(1,51 \%)$ no Serra Dourada, entre outros, de acordo com a Figura 3. Cabe ressaltar que não houve preferência por classe econômica, considerando que os bairros Vila Aurora, Centro e Santa Cruz são aqueles com maior renda média do município, enquanto os demais são enquadrados como bairros de periferia e que apresentam menor índice de renda. quinquterios $2007-2011$ e 2012 -2016.

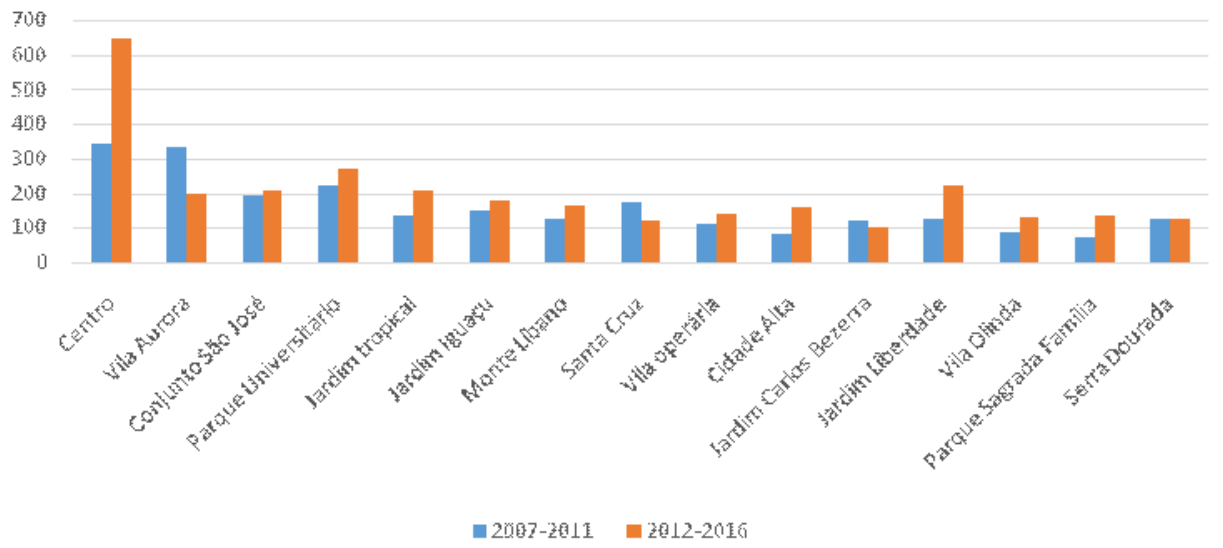

Figura 3 - Distribuição comparativa de casos notificados de dengue por bairro entre os anos 2007 a 2011 e 2012 a 2016, segundo os dados do SINAN. Rondonópolis, MT, Brasil, 2017 
Na Figura 3, também é possível observar o aumento do total de número de casos em comparação do período de 2007-2011 ao quinquênio 2012-2016 em alguns bairros, como o Cidade Alta com elevação de 75 casos (89,28\%) e o Jardim Tropical com aumento de 70 casos $(51,47 \%)$. Em outros bairros houve redução dos casos, como no Vila Aurora com 139 casos a menos (41,49\%) de casos e o Santa Cruz com $53(30,63 \%)$.

\section{DISCUSSÃO}

Nesta pesquisa, o total de casos aumentou em 16,87\% entre os anos 2012 a 2016 se comparado os anos de 2007-2011, sendo que em 2010 houve o maior registro de casos 4153 (24,94\%). Nos anos de 2000 a 2015 , verificou-se o aumento de $232,7 \%$ no número total de casos de dengue no Brasil, em todo seu território ${ }^{(13)}$.

No Município de Santos-SP, um estudo retrospectivo descritivo de dengue entre 2009 e 2013 apontou 18.918 casos notificados, sendo que 9.812 (51,87\%) ocorreram em 2013 e 8.280 (43,77\%) em 2010 ${ }^{(14)}$. Nos anos 2008, 2009 e 2010, foram registrados 3.313 casos positivos no município de Colíder-MT, sendo o menor índice 175 em 2010 e a maior quantidade 2.616 em 2009, o qual se diferenciou dos demais anos com aumento exponencial, destacando-se a temporalidade na distribuição dos casos nesse ano ${ }^{(15)}$. As características do ambiente favorecem a proliferação do vetor, logo são diversos fatores, entre eles ambientais e sociais, que se correlacionam com o aumento da reprodução e disseminação do vetor e da doença.

O perfil sociodemográfico das notificações de dengue em Rondonópolis caracterizou-se por predominarem casos do sexo feminino, faixa etária adulta de 31 a 54 anos e raça branca. O predomínio da alta taxa da doença entre as mulheres está relacionado com a maior permanência no domicílio, local preferido pelo mosquito Aedes aegypti e pela característica urbana do vetor ${ }^{(16)}$.

Resultados semelhantes foram encontrados entre 2007 a 2011 em Cuiabá-MT com maior notificação de casos no sexo feminino $(53,0 \%)$, porém predominou a faixa etária entre 5 a 14 anos $(25,1 \%)^{(8)}$. No entanto, esta doença não está ligada ao sexo, logo se encontram resultados divergentes, como o estudo $^{(10)}$ sobre o perfil clínico-epidemiológico em Juscimeira-MT entre os anos de 2009 a 2013, com predomínio 201 (51,4\%) do sexo masculino.

Quanto à raça, em Juiz de Fora-MG, os indivíduos brancos predominaram nos casos registrados no ano de 2010, com 25,7\%, porém 61,9\% dos casos registrados tiveram esse fator ignorado ${ }^{(17)}$. Semelhantemente, em Rondonópolis foi encontrado um elevado percentual de ocorrência de etnia ignorada, provavelmente pelo fato da classificação dos indivíduos em relação à cor ser considerada uma variável subjetiva, o que dificulta o preenchimento adequado da notificação.

Quanto à variável escolaridade, também não foi preenchida a maioria das notificações 9244 (55,51\%) e predominou a zona de residência urbana 16018 (96,20\%). A subnotificação das doenças infectocontagiosas, incluindo a variável nível de escolaridade, é umas das questões que devem ser discutidas pelos profissionais e gestores em saúde, considerando a importância do conhecimento destes para o preenchimento correto dos dados, a fim de contribuir com medidas de intervenção e controle da dengue ${ }^{(18)}$.

O vetor da dengue se tornou predominantemente da zona urbana, tal como se registrou nesta pesquisa, por sua forte relação aos objetos manufaturados pelo homem que servem como criadouros, não exigindo associação socioeconômica e ambiental. Porém, seus focos são normalmente encontrados em lugares no qual há precariedade da limpeza pública, acúmulos de resíduos sólidos, falhos sistemas de drenagem, condições de saneamento precárias, deslocamento da população de forma não planejada, quantidades de pessoas residentes em um mesmo domicílio e seu nível de escolaridade, além de possíveis utensílios encontrados nas casas como: tambor, tanque, tina, caixa d'água, vasos de plantas, cisterna, cacimba, entre outros ${ }^{(19)}$.

Além disso, os municípios que apresentam a maior quantidade dos casos de dengue são os que possuem maior concentração populacional, como é o caso de Rondonópolis, considerando que a aglomeração influencia a interação entre o vetor, o vírus e o homem. ${ }^{(9)}$ Os meses de maior notificação foram janeiro, fevereiro e março, considerado o período chuvoso, que contribui com o aumento da 
longevidade do Aedes aegypti e a possibilidade de transmissão da doença. Em Campo Grande-MS, nos anos de 2001, 2002, 2007 e 2010, a incidência da dengue ocorreu de janeiro a julho, porém com início moderado nos meses de novembro e dezembro ${ }^{(20)}$.

É importante destacar que esta pesquisa revelou casos diagnosticados de dengue clássica e hemorrágica, demonstrando a gravidade das ocorrências e o risco de morte pela doença. É importante destacar que a dengue clássica apresenta-se primeiramente com febre de $39^{\circ} \mathrm{C}$ a $40^{\circ} \mathrm{C}$, associada à dor de cabeça, nas articulações e no corpo e fraqueza; na hemorrágica, observa-se o surgimento de manifestações hemorrágicas espontâneas ou provocadas, pela diminuição de plaquetas no sangue e perda de plasma para o terceiro espaço, sendo esta última de maior gravidade. O risco do indivíduo apresentar dengue hemorrágica é maior quando a segunda infecção é causada pelo DEN-2. A evolução de dengue clássica para hemorrágica ocorre em pequena percentagem de indivíduos (0,3\% a 4\%). Nos períodos epidêmicos, até $40 \%$ dos infectados podem apresentar a forma assintomática, só identificada por meio da viragem sorológica específica com anticorpos IgM e IgG antivírus da dengue ${ }^{(21)}$.

Em uma pesquisa que descreveu o perfil clínico-epidemiológico dos casos de dengue no estado do Paraná, houve um crescimento alarmante de $6.000 \%$ nos casos de óbito no biênio de 2015/2016 em relação a 2011/2012, observado também pela incidência dos casos de 22,99/100.000 hab em 2011/2012 para 472,17/100.000 hab em 2015/2016 ${ }^{(22)}$. Nos últimos dez anos, registraram-se em Goiás mais de 300 mil casos de dengue, com destaque para o ano de 2011 e em 2013, até a primeira quinzena do mês de novembro, havia cerca de 156.620 notificações, e destes, 65 óbitos foram confirmados $^{(23)}$.

No que tange à localização dos casos notificados, o endereço constitui-se a única referência geográfica no banco de dados do SINAN, observando-se uma distribuição por toda a extensão territorial de Rondonópolis, tornando-se possível a identificação dos bairros com maior número de focos da doença: na região central, norte e oeste do município. Vários fatores contribuem para proliferação do vetor e para a transmissão do vírus, entre estes, o crescimento da população associado com a urbanização não planejada e descontrolada, principalmente em países com clima tropical que criam condições ideais para a proliferação do Aedes aegypti(24).

Entender que a concentração populacional influencia na incidência elevada da dengue é fundamental para explicar o número elevado de casos, sendo possível ser associada às condições precárias de saneamento, moradia, educação e fatores culturais. Mas é preciso ressaltar que a população mais carente vive em piores condições sociais, ambientais e sanitárias, assim como menor acesso à saúde em particular, o que pode contribuir para as ocorrências da doença ${ }^{(24-25)}$.

Considerando que a maioria dos casos registrados ocorreu nas áreas centrais (Centro e Vila Aurora), é importante destacar que estes bairros não possuem cobertura de saúde dos usuários cadastrados e acompanhados pela Estratégia de Saúde da Família (ESF), local de desenvolvimento de ações de prevenção e controle da doença. Sugere que, para o controle desta epidemia, sejam realizadas medidas preventivas pelos profissionais de saúde, além da contratação de equipe capacitada pelos gestores para desenvolver o serviço de detecção de larvas para o combate e conscientização da população do município, com enfoque e prioridade nos bairros mais populosos ${ }^{(25)}$. Outras características que dificultam o combate à dengue em metrópoles são a falta de conhecimento da população e a compreensão do seu papel social nesse âmbito, além da dificuldade de acesso às residências pelos agentes de controle do vetor e entre outros ${ }^{(24)}$.

Um fato destacado em Rondonópolis-MT é que alguns dos bairros populosos, com maior registro de casos identificados no mapa, possuem condições precárias de infraestrutura e baixo poder socioeconômico como a Vila Olinda, e outros possuem melhores condições como o Centro e Vila Aurora. Também é possível ressaltar no presente estudo que os bairros citados nesta pesquisa são próximos a rios, córregos e áreas sob proteção ambiental, conforme analisado no mapa do município.

A limitação deste estudo refere-se ao fato que alguns bairros não constam no shapefile do Município, considerando as alterações realizadas pelos gestores e os bairros notificados pelos profissionais e que são exportados para o sistema de informação à saúde. Além disso, torna-se necessário o preenchimento adequado dos campos disponíveis nas fichas, para se evitar a subnotificação dos bairros e a adequação do sistema, impedindo que ocorram falhas no cadastro dos casos notificados. Desta forma, sugere-se a intensificação de educação permanente em saúde sobre a notificação dos casos para aqueles que manipulam os dados na atenção primária à saúde. 
Esta pesquisa tornou possível a identificação dos focos, mostrando o retrato geográfico da dengue, no intuito de alertar os pesquisadores e a gestão de saúde do município o comportamento da doença, além de propor e favorecer o desenvolvimento de ações voltadas para a prevenção, controle e eliminação dos casos.

Através do mapeamento dos casos de dengue e pelo conhecimento da transmissibilidade em áreas de abrangência, pode-se ter acesso eficiente às informações necessárias para realizar a vigilância dos casos e criar condições para avaliar a necessidade da descentralização dos tratamentos por meio de ações educativas para a população e realocação dos recursos humanos e físicos. Por fim, sugere-se que novos estudos sejam realizados a fim de incluírem outras variáveis associadas à ocorrência dos casos de dengue.

\section{REFERÊNCIAS}

1. Lopes N, Nozawa C, Linhares REC. Características gerais e epidemiologia dos arbovírus emergentes no Brasil. Rev. Pan-AmazonicaSaud[Internet]. 2014 [acesso em 2018 abr25];5(3). Disponívelem:http://scielo.iec.gov.br/pdf/ rpas/v5n3/v5n3a07.pdf.

2. Secretaria do Estado de Saúde de São Paulo. Diretrizes para a prevenção e controle das arbovirosesurbanas no estado de São Paulo. [Internet]. 2017 [acesso em 2018 abr25]. Disponível em: http://www.saude.sp.gov.br/ resources/cve-centro-de-vigilancia-epidemiologica/publicacoes/diretrizes2017_arboviroses_esp.pdf.

3. World Health Organization (WHO). Dengue vaccine. WHO position paper. Geneva: WHO; 2016.

4. Terra MR, Silva RS, Pereira MGN, Lima AF. Aedes aegypti e as arbovíroses emergentes no Brasil. Rev. Uninga[Internet]. 2017 [acesso em 2018 abr25];30(3). Disponível em: http://revista.uninga.br/index.php/ uningareviews/article/view/2028.

5. Picinato MAC, Grisolio APR, Caselani K, Nunes JOR, Carvalho AAB, Ferraudo AS. Dengue: uma visão sobre o vetor urbano Aedes aegypti e a difícil interface do seu controle. vet. em Foco. [Internet]. 2015 [acesso em 2018 abr25];13(1) Disponível em: http://www.periodicos.ulbra.br/index.php/veterinaria/article/view/2132/1982.

6. Viana DV,Ignotti E. A ocorrência da dengue e variações meteorológicas no Brasil: revisão sistemática. Rev. bras. epidemiol. [Internet]. 2013 [acesso em 2017 fev24];16(2). Disponível em: http://dx.doi.org/10.1590/S1415790X2013000200002.

7. Bessa Junior FN, Nunes RFF, de Souza MA, de Medeiros AC, Marinho MJM, Pereira WO. Spatialdistributionof dengue disease in municipality of Mossoró, Rio Grande do Norte, using the Geographic Information System. Rev. bras. epidemiol.[Internet]. 2013 [acesso em 2015 dez 15];16(3). Disponível em: http://dx.doi.org/10.1590/ S1415-790X2013000300005.

8. Guollo DSO, Silva RC,Peluzio JM, Carmo Filho JR. Aspectos epidemiológicos da dengue em Cuiabá-MT entre 2007 e 2011. Estudos [Internet]. 2015 [acesso em 2017 mar 23];42(4) Disponível em: http://seer.pucgoias.edu.br/ index.php/estudos/article/viewFile/4368/2503.

9. Fernandes RS, Neves SMAS, Neves RJ. Risco da dengue nos municípios pantaneiros de Mato Grosso, Brasil. Bol Goiano Geogr[Internet]. 2014 [acesso em 2017 jan22];34(1). Disponível em: https://doi.org/10.5216/bgg. v34i1.29317.

10. Assunção ML, Aguiar AMM. Perfil clínico-epidemiológico da dengue no município de Juscimeira - MT. RevEpidemiolControllnfect. [Internet]. 2014 [acesso em 2017 nov 06];4(4). Disponível em: http://dx.doi. org/10.17058/reci.v4i4.5605.

11. Instituto Brasileiro de Geografia e Estatística (IBGE). Estimativa de População 2014. [Internet]. 2014 [acesso em 2017 fev06]. Disponível em: http://cidades.ibge.gov.br/xtras/perfil.php?codmun=510760.

12. Prefeitura do município de Rondonópolis. Atualização do plano diretor municipal, leitura técnica 
socioterritorial. Plano diretor e revisão da legislação urbanística. Rondonópolis: Prefeitura do município de Rondonópolis; 2017. 281 p. Relatório técnico.

13. Araújo VEM,Bezerra JMT,Amâncio FF,Passos VMA,Carneiro M. Increase in the burden of dengue in Brazil and federated units, 2000 and 2015: analysis of the Global Burden of Disease Study 2015. Rev. bras. epidemiol. [Internet]. 2017 [acesso em 2017 jun04];1(20). Disponível em: http://dx.doi.org/10.1590/1980-5497201700050017.

14. Cruz CA, Paula EMN, Nogueira CSL, Meirelles-Bartoli RB, Carvalho AAB. Dengue em São Paulo/SP: um estudo retrospectivo dos casos entre 2009 e 2013. ARS Veterinária [Internet]. 2015 [acesso em 2017 jan12];31(2). Disponível em: http://dx.doi.org/10.15361/2175-0106.2015v31n2p37.

15. Cavalheiro MA. Análise da prevalência de dengue no Município de Colíder-MT nos anos de 2008 a 2010. FACIDER Rev.Cient.[Internet]. 2015 [acesso em 2017 mar 23];(7). Disponível em: http://sei-cesucol.edu.br/revista/ index.php/facider/article/view/121.

16. Gibson G, Souza-Santos R, Brasil P, Pacheco AG, Cruz OG, Honório NA, et. al.From primary care to hospitalization: clinical warning signs of severe dengue fever in children and adolescents during an outbreak in Rio de Janeiro, Brazil. CadSaude Publica [Internet]. 2013 [acesso em 2017 maio 06];29(1). Disponível em: https:// www.arca.fiocruz.br/handle/icict/10528.

17. Assis VC, Amaral MPH, Mendonça AE. Análise da qualidade das notificações de dengue informadas no sistema de informação de agravos de notificação, na epidemia de 2010, em uma cidade polo da zona da mata do estado de Minas Gerais. Rev APS [Internet]. 2014 [acesso em 2018 abr25];17(4). Disponível em: https://aps.ufjf.emnuvens. com.br/aps/article/view/2103.

18. Silva GA, Oliveira CMG. O registro das doenças de notificação compulsória: a participação dos profissionais da saúde e da comunidade. RevEpidemiolControlInfect. [Internet]. 2014 [acesso em 2016 ago23];(4):215-20. Disponível em: http://dx.doi.org/10.17058/reci.v4i3.4578.

19. Araujo MR, Desmoulière SJM, Levino A. Padrão espacial da distribuição da incidência de dengue e sua relação com a variável renda na Cidade de Manaus, Estado do Amazonas, Brasil. Rev Pan-Amaz Saude[Internet]. 2014 [acesso em 2017 jan21];5(2) Disponível em: http://scielo.iec.gov.br/pdf/rpas/v5n2/v5n2a02.pdf.

20. Pacheco ICS, Carvalho AMA, Ponte ERJC, Silva MG. Relação entre condições climáticas e incidência de dengue no Município de Campo Grande, MS. Multitemas. [Internet]. 2017 [acesso em 2016 jun15];22(51). Disponível em: http://dx.doi.org/10.20435/multi.v22i51.1123.

21. Dalbem AG, Herling JD, Vieira RG, Souza AI. Dengue clássica e febre hemorrágica da dengue: etiologia, fisiologia, epidemiologia e fatores de risco. RevCiênEstudAcadMed[Internet] 2014 [acesso em 2017 jul10];(1). Disponível em: http://www.unemat.br/revistas/revistamedicina/media/files/dengue_classica.pdf.

22. Brigadão GS, Corrêa NAB. Levantamento epidemiológico da dengue no estado do Paraná Brasil nos anos de 2011 a 2015. Arq. ciências saúde UNIPAR[Internet]. 2017 [acesso em 2017 jul10];21(1). Disponível em: https://doi. org/10.25110/arqsaude.v21i1.2017.6075.

23. Cançado MSM, Oliveira ESF, Teixeira RAG. Educação em saúde para prevenção e controle da dengue: percepção de representantes de um comitê de mobilização contra dengue de uma região do centro-oeste do Brasil. Invest Qual Educ [Internet]. 2014 [acesso em 2017 jun10];2(1). Disponível em: http://proceedings.ciaiq.org/ index.php/CIAIQ/article/viewFile/523/518.

24. Johansen $\mathrm{Cl}$, Carmo RL, Alves LC. Desigualdade social intraurbana: implicações sobre a epidemia de dengue em Campinas, SP, em 2014. Cad. Metrop. [Internet] 2016 [acesso em 2018 abr25];18(36). Disponível em: http:// dx.doi.org/10.1590/2236-9996.2016-3606.

25. Paula FR, Delmondes PH, Roewer SP, Nascimento MVM, Scherer EF. Prevalência dos casos de dengue no Município de Barra do Garças - MT, no período de dezembro de 2008 à dezembro de 2012. Interdisciplinar: Interdiscip. Rev. Eletrônica UNIVAR [Internet] 2014 [acesso em 2017 mar 28];1(11). Disponível em: https://updoc. site/download/prevalencia-dos-casos-de-dengue-no-municipio-de-barra-do_pdf. 